Proceedings of the 45th International School and Conference on the Physics of Semiconductors "Jaszowiec" 2016, Szczyrk

\title{
Optical Characterization of ZnO Nanorods Grown by the Ultra-Fast and Low Temperature Hydrothermal Process
}

\author{
B.S. Witkowski ${ }^{a, *}$, V.Y. IVAnOV ${ }^{a}$, Ł. WAChnicki ${ }^{a}$, S. GieraŁtowska $^{a}$ \\ AND M. GODLEWSKI ${ }^{a, b}$ \\ ${ }^{a}$ Institute of Physics, Polish Academy of Sciences, Aleja Lotnikow 32/46, PL-02668 Warsaw, Poland \\ ${ }^{b}$ Dept. of Mathematics and Natural Sciences College of Science, Cardinal S. Wyszyński University, \\ Dewajtis 5, 01-815 Warsaw, Poland
}

\begin{abstract}
The results of photoluminescence investigations of zinc oxide nanorods are reported. These nanorods grown on undoped silicon substrates were obtained by low temperature and ultra-fast version of a microwave-assisted hydrothermal method. The photoluminescence investigations show very high quality of the obtained material From photoluminescence studies we conclude the lack of carrier localization effects. The photoluminescence is dominated by band gap edge emission of bound excitonic (donor bound excitons) origin. Thus, the photoluminescence quenching observed at increased temperatures is associated with thermal ionization of shallow donors. From photoluminescence analysis (changes of photoluminescence line width) a strength of exciton-acoustic phonon coupling is evaluated.
\end{abstract}

DOI: $10.12693 /$ APhysPolA.130.1199

PACS/topics: 78.67.-n, 61.46.+w, 81.10.Dn, 77.84.Bw

\section{Introduction}

Zinc oxide is a II-VI semiconductor, which has a direct energy gap of about $3.37 \mathrm{eV}$ at room temperature [1] and high exciton binding energy $(60 \mathrm{meV})$. High transparency and high $n$-type conductivity allow application of $\mathrm{ZnO}$ as an electrode in solar cells [2]. $\mathrm{ZnO}$ was also tested for other applications, e.g. as an active part of transparent transistors [3], material for the construction of photovoltaic cells [4], detectors [5] and optoelectronic devices [6]. For most of these applications it is crucial to understand optical properties of $\mathrm{ZnO}$ nanostructures.

In recent works we developed a new method of a growth of monocrystalline $\mathrm{ZnO}$ nanorods (NRs) [7]. The nanorods are grown at very low temperature and at very short time (say $3 \mathrm{~min}$ ) using inexpensive precursors. In the present work we discuss properties of these nanorods based on the results of photoluminescence (PL) and PL temperature-dependence measurements. Role of local potential fluctuations, excitons localization effects and phonon-exciton coupling is investigated.

\section{Experimental details}

An ultra-fast growth of $\mathrm{ZnO}$ nanorods was possible by modification of a microwave assisted hydrothermal method. Importantly (for range of possible industrial applications) it is safe and cheap growth method. The detail description of the growth process is given in Ref. [7]. As a substrate undoped $\mathrm{Si}(1 \mathrm{~cm} \times 1 \mathrm{~cm}$, orientation: 100)

\footnotetext{
${ }^{*}$ corresponding author; e-mail: bwitkow@ifpan.edu.pl
}

was used. First, a nucleation layer for the hydrothermal growth was created through 10 cycles of atomic layer deposition (ALD) process, using water and diethyl zinc as oxygen and zinc precursors, respectively. Then such prepared substrate was moved to a hydrothermal reactor. NRs of zinc oxide were grown from the solution, using a zinc acetate dihydrate, sodium hydroxide, and isopropanol purchased from Sigma-Aldrich Co. The reaction mixtures were prepared by dissolving zinc acetate in deionized water, and then precipitating with 1-mol solution of sodium hydroxide to the $\mathrm{pH} 8$. Several series of NRs of a length close to $500 \mathrm{~nm}$ and a different width were grown by manipulating concentration of a zinc precursor in a water solution. Zinc concentrations of 0.1 $(\approx 50 \mathrm{~nm}$ width $), 0.15(\approx 120 \mathrm{~nm})$ and $0.2 \mathrm{~mol} / \mathrm{dm}^{3}$ $(\approx 200 \mathrm{~nm})$ were used. The solution with the prepared substrate was heated to $40^{\circ} \mathrm{C}$ and held at that temperature for $2 \mathrm{~min}$ in the microwave hydrothermal reactor ERTEC Magnum II. After the growth process, the samples were washed with isopropanol and dried in air.

Photoluminescence measurements have been performed in the temperature range of 6-300 K, using a multipurpose optical spectrometer based on double grating spectrograph $(F=500 \mathrm{~mm})$ equipped with TE cooled CCD camera.

\section{Results and discussion}

Photoluminescence is one of the basic methods for evaluation the samples quality. The study of PL (origin of PL lines, their spectral position and width of the excitonic lines, etc.) and its temperature dependence yields an important information on samples quality. The PL analysis allows determination of the presence of the carriers localization effects [8], coupling of excitons with the 
acoustic and optical phonons and temperature stability of the PL [9]. In the present study we verify our recent statement that NRs, grown by the innovative microwaveassisted hydrothermal method, are characterized by high quality $[7,10]$.

The first result which supports the above statement is that PL of ZnO NRs can be observed only at UV spectral region. Only band edge PL is observed, dominated by excitonic transitions. No deep defects-related emission was observed. The band edge PL of our nanorods is shown in Fig. 1. Dominant peak at $3.364 \mathrm{eV}$ is due to superposition of donor bound excitons lines and weak band-toband emission. The observed PL is compared with the one observed for high quality bulk material (see Fig. 1).

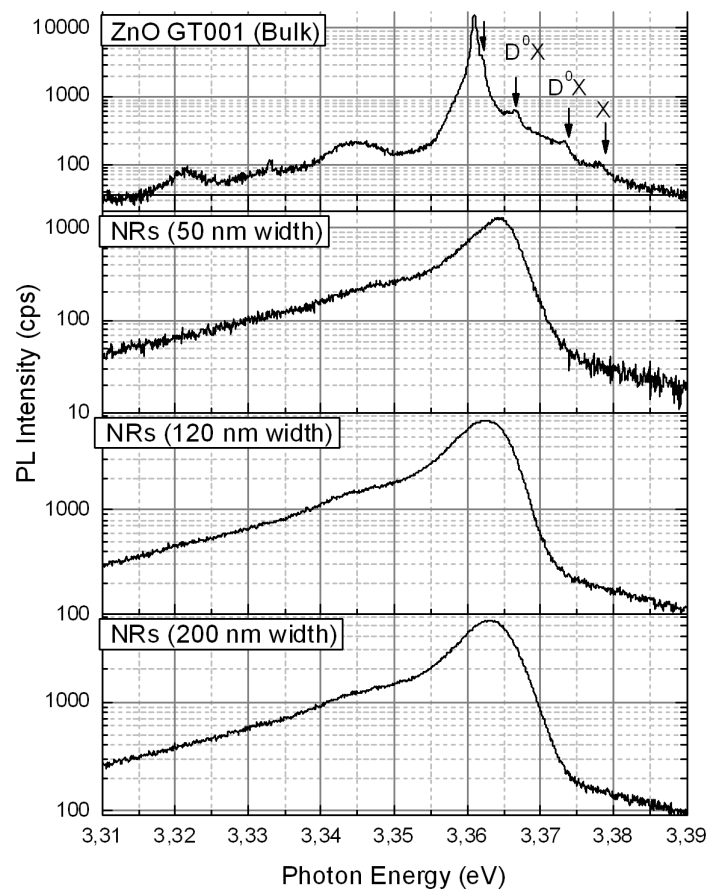

Fig. 1. PL spectra for series of NRs of a different width compared to PL of high quality $\mathrm{ZnO}$ bulk sample. Temperature $5 \mathrm{k}, 3.807 \mathrm{eV}$ excitation.

In Fig. 2 a fit to the PL is shown. The observed asymmetric shape of the PL can be explained by superposition of $4 \mathrm{PL}$ peaks with a Gaussian line shape. Fit perfectly reproduces the experimental result. We attribute the dominant component to donor bound excitonic (DBE) transition, whereas the one at $3.345 \mathrm{eV}$ - to LO replica of excitonic emission.

From the fit we could decompose the PL spectrum and estimate full width at half maximum (FWHM) of each of the contributing $\mathrm{PL}$ lines, in particular the dominant $3.364 \mathrm{eV}$ band of the DBE origin. The FWHM, which depends on the $\mathrm{ZnO}$ NRs sizes, is $5.0 \mathrm{meV}$ for NRs of $50 \mathrm{~nm}$ width, $6.7 \mathrm{meV}$ for NRs of $120 \mathrm{~nm}$ width and $8.2 \mathrm{meV}$ for NRs of $200 \mathrm{~nm}$ width. The value of $5 \mathrm{meV}$ of FWHM is comparable with the one reported for high quality $\mathrm{ZnO}$ samples [11].

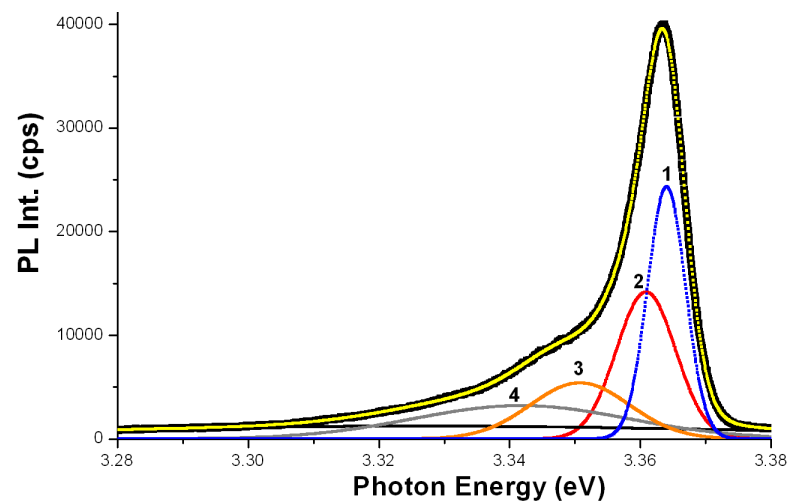

Fig. 2. $\mathrm{ZnO}$ NRs PL spectra for nanorods of $50 \mathrm{~nm}$ width taken at temperature of $6 \mathrm{~K}$. Fit with 4 Gaussian peaks reproduces the PL line shape. With the black line experimental data are shown, yellow points show area superposition of four Gaussian peaks.

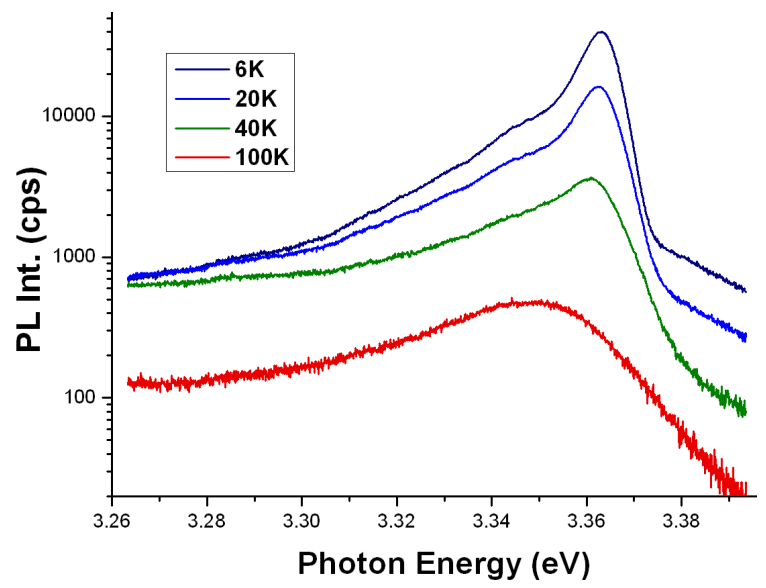

Fig. 3. ZnO NRs of $120 \mathrm{~nm}$ width PL spectra collected at different temperatures.

A strong temperature quenching of the DBE PL was observed. At temperature above $100 \mathrm{~K}$ band DBE excitonic emission is replaced by free-to-band and/or donoracceptor pair emission. Whole PL is then deactivated, as is shown in Fig. 3 for temperature range 1 to $100 \mathrm{~K}$.

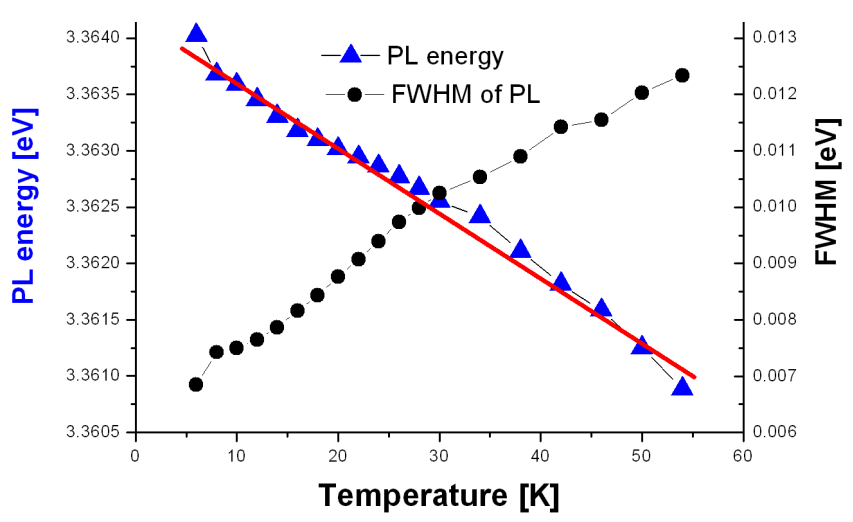

Fig. 4. Energy position of the DBE PL peak and its FWHM in function of temperature. 
Using the convolution procedure shown in Fig. 2, we determined also temperature dependences of all four PL bands obtained from line decomposition. We followed their energy position and FWHM. Such an analysis is commonly used to evaluate role of localization effects in samples [8]. If localization effect are important, S-shape temperature-dependence should be observed. The results obtained in present work for the DBE line are shown in Fig. 4.

The S-shape dependence is not observed and the FWHM is linearly increasing with temperature, as expected for exciton-phonon coupling in high quality samples.

The FWHM can be approximately described by the following equation:

$$
\Gamma(T)=\gamma_{\mathrm{inh}}+\gamma_{\mathrm{ph}} T+\Gamma_{\mathrm{LO}} /\left[\exp \left(\hbar \omega_{\mathrm{LO}} / k_{\mathrm{B}} T\right)-1\right],
$$

where $\gamma_{\text {inh }}$ is a temperature-independent term that denotes the inhomogeneous linewidth due to the excitonexciton, exciton-carrier interactions [9], and the scattering by defects, impurities. The second term $\gamma_{\mathrm{ph}} T$ is due to acoustic phonon scattering. This term represents the acoustic phonon coupling strength, mainly caused by a deformation potential mechanism. The third term describes scattering due to optical phonons. At low temperatures, the line width increases from the zero-temperature value first because of the scattering by acoustic phonons. Note that the population of LO phonon is vanishingly small over this temperature range.

By fitting to the results shown in Fig. 4, we estimated the $\gamma_{\text {inh }}$ value, which is equal to $6.4 \mathrm{meV}$ and $\gamma_{\mathrm{ph}}$ is equal to $115 \mathrm{\mu eV} / \mathrm{K}$. For example, for MBE grown $\mathrm{ZnO}$-based quantum-well system the relevant values were $\gamma_{\text {inh }}=17 \mathrm{meV}$ and $\gamma_{\mathrm{ph}}=31 \mathrm{\mu eV} / \mathrm{K}$ [12]. Thus, we can claim that much smaller inhomogeneous broadening is observed in our case, confirming very reduced role of disorder and localization effects in our NRs.

The temperature dependence of the PL energy position can be described by the Varshni formula [13] or by the following Bose-Einstein expression [14]:

$$
E(T)=E(0)-\lambda /[\exp (\beta / T)-1],
$$

where $\lambda, \beta$ are fitting parameters, and $E(0)$ is the band gap at a temperature of $0 \mathrm{~K}$.

Also here, when localization effects are not important, the above dependence can be approximated by a linear one explaining the date shown in Fig. 4.

\section{Summary}

A high quality of the $\mathrm{ZnO} \mathrm{NRs}$ is confirmed by observation of intensive band edge emissions of bound excitonic origin. Importantly deep defect related emissions are not observed. Influence of local potential fluctuations on emission bands, was not observed. From temperature-dependence of the FWHM peak we estimated the strength of exciton-acoustic phonon coupling, which is significantly larger, than in the case of structures with high localization effects.

\section{Acknowledgments}

The research was partially supported by the Polish National Science Centre (NCN) - UMO2012/06/A/ST7/00398.

\section{References}

[1] C. Klingshirn, Phys. Status Solidi B 244, 3027 (2007).

[2] M. Godlewski, E. Guziewicz, G. Łuka, T. Krajewski, M. Łukasiewicz, Ł. Wachnicki, A. Wachnicka, K. Kopalko, A. Sarem, B. Dalati, Thin Solid Films 518, 1145 (2009).

[3] S. Gieraltowska, L. Wachnicki, B.S. Witkowski, M. Godlewski, E. Guziewicz, Thin Solid Films 520, 14 (2012).

[4] R. Pietruszka, B.S. Witkowski, S. Gieraltowska, P. Caban, L. Wachnicki, E. Zielony, K. Gwozdz, P. Bieganski, E. Placzek-Popko, M. Godlewski, Solar Energy Mater. Solar Cells 143, 99 (2015).

[5] B.S. Witkowski, L. Wachnicki, S. Gieraltowska, P. Sybilski, K. Kopalko, M. Stachowicz, M. Godlewski, Phys. Status Solidi C 11, 9 (2014).

[6] U. Ozgur, D. Hofstetter, H. Morkoc, Proc. IEEE 98, 7 (2010).

[7] B.S. Witkowski, L. Wachnicki, S. Gieraltowska, P. Dluzewski, A. Szczepanska, J. Kaszewski, M. Godlewski, Int. J. Nanotechnol. 11, 758 (2014).

[8] Q. Li, S.J. Xu, M.H. Xie, S.Y. Tong, J. Phys. Condens. Matter 17, 30 (2005).

[9] S. Rudin, T.L. Reinecke, B. Segall, Phys. Rev. B 42, 11218 (1990).

[10] B.S. Witkowski, L. Wachnicki, S. Gieraltowska, A. Reszka, B.J. Kowalski, M. Godlewski, Microsc. Microanal. 21, 3 (2015).

[11] Y. Chen, D.M. Bagnall, H. Koh, K. Park, K. Hiraga, Z. Zhu, T. Yao, J. Appl. Phys. 84, 3912 (1998).

[12] H.D. Sun, T. Makino, N.T. Tuan, Y. Segawa, M. Kawasaki, A. Ohtomo, K. Tamura, H. Koinuma, Appl. Phys. Lett. 78, 2464 (2001).

[13] Y.P. Varshni, Physica 34, 1 (1967).

[14] P. Lautenschlager, M. Garriga, S. Logothetidis, M. Cardona, Phys. Rev. B 35, 9174 (1987). 